\title{
Efficacy of pembrolizumab in patients with pituitary carcinoma: report of four cases from a phase II study
}

\author{
Nazanin Majd (D) , ${ }^{1}$ Steven G Waguespack, ${ }^{2}$ Filip Janku, ${ }^{3}$ Siqing Fu, ${ }^{3}$ \\ Marta Penas-Prado, ${ }^{1}$ Mingxuan Xu, ${ }^{3}$ Anas Alshawa, ${ }^{3}$ Carlos Kamiya-Matsuoka, ${ }^{1}$ \\ Shaan M Raza, ${ }^{4}$ Ian E McCutcheon, ${ }^{4}$ Aung Naing (D ${ }^{3}$
}

To cite: Majd N,

Waguespack SG, Janku F, et al. Efficacy of pembrolizumab in patients with pituitary carcinoma: report of four cases from a phase II study. Journal for ImmunoTherapy of Cancer 2020;8:e001532. doi:10.1136/ jitc-2020-001532

NM and SGW are Co-first authors.

Accepted 15 November 2020

Check for updates

(C) Author(s) (or their employer(s)) 2020. Re-use permitted under CC BY-NC. No commercial re-use. See rights and permissions. Published by BMJ.

${ }^{1}$ Department of Neuro-Oncology, The University of Texas MD Anderson Cancer Center, Houston, Texas, USA

${ }^{2}$ Department of Endocrine Neoplasia and Hormonal Disorders, The University of Texas MD Anderson Cancer Center, Houston, Texas, USA

${ }^{3}$ Department of Investigational Cancer Therapeutics, University of Texas at MD Anderson Cancer Center, Houston, Texas, USA

${ }^{4}$ Department of Neurosurgery, The University of Texas MD Anderson Cancer Center, Houston, Texas, USA

Correspondence to

Dr Aung Naing;

anaing@mdanderson.org

\section{ABSTRACT}

Pituitary carcinoma is an aggressive tumor characterized by metastatic spread beyond the sellar region. Symptoms can be debilitating due to hormonal excess and survival is poor. Pituitary carcinomas recur despite conventional multimodality treatments. Given the recent advances in the use of immune checkpoint inhibitors (CPIs) to treat various solid cancers, there has been interest in exploring the role of immunotherapy for treating aggressive, refractory pituitary tumors. We treated 4 patients with pituitary carcinoma with pembrolizumab as part of a phase II clinical trial. Two patients (patients 1 and 2) with functioning corticotroph pituitary carcinomas (refractory to surgery, radiotherapy and chemotherapy) had partial radiographic (60\% and $32 \%$ per Immune-Related Response Evaluation Criteria In Solid Tumors, respectively) and hormonal responses. Patient 1's response continues 42 months after initiation of pembrolizumab and his tumor tissue obtained after treatment with temozolomide demonstrated a hypermutator phenotype with $M S H 2$ and MSH6 gene mutations. Patient 2's tumor after exposure to temozolomide was not sampled, but prior somatic mutational testing was negative. One patient with a non-functioning corticotroph tumor (patient 3 ) had a best response of stable disease for 4 months. One patient with a prolactin-secreting carcinoma (patient 4) had progressive disease. The latter 2 patients' tumors did not demonstrate a hypermutator phenotype after treatment with temozolomide. Programmed death-ligand 1 staining was negative in all tumors. We report 2 cases of corticotroph pituitary carcinoma responsive to pembrolizumab after prior exposure to alkylating agents. The role of CPIs in treating patients with pituitary carcinoma, the relationship between tumor subtype and response to immunotherapy and mechanisms of hypermutation in this orphan disease require further study. Trial registration number: NCT02721732.

\section{INTRODUCTION}

Pituitary carcinoma (PC) is defined as a pituitary adenoma that has metastasized outside the sellar region. ${ }^{1}$ Unlike pituitary adenomas that are common and usually benign, ${ }^{2}$ PCs are aggressive, rare tumors that account for an estimated 200-300 cases annually in the USA. $^{3}$
Similar to pituitary adenomas, PCs originate from the various cell types within the anterior pituitary gland and can be functioning (hormone secreting) or nonfunctioning. PCs more often are lactotroph or corticotroph tumors, producing prolactin (PRL) or adrenocorticotropic hormone (ACTH), respectively. These tumors can be clinically apparent, causing signs and symptoms of hormone excess, or they can be clinically silent, demonstrating expression of PRL or ACTH via immunohistochemistry but not causing Cushing syndrome or clinical features of hyperprolactinemia.

Given the rarity of PC, no well-defined standard therapy exists. Most patients undergo resection if feasible, focal radiotherapy, and medical therapy (for functioning tumors). Temozolomide has emerged as a first-line chemotherapy for PC. ${ }^{4}$ The combination of temozolomide with capecitabine (CAPTEM) has also been shown to result in high response rates and prolonged survival in $\mathrm{PC}^{5}$ However, prolonged use of temozolomide can be associated with bone marrow suppression, and recurrences are common in clinical practice. With the advent of immunotherapy as a fourth pillar for the treatment of various solid malignancies, there has been interest in exploring the role of checkpoint inhibitors (CPIs) for treatment of pituitary tumors.

CPIs, including antibodies against programmed death-1 (PD-1), programmed death-ligand 1 (PD-L1) and cytotoxic T-lymphocyte-associated protein 4 (CTLA-4), significantly prolong survival in $30 \%-40 \%$ of patients with various solid cancers. ${ }^{6}$ Mutation status, PD-L1 expression and the presence of tumor-infiltrating lymphocytes (TILs) are potential biomarkers of response to CPIs. ${ }^{6}$ Different thresholds have been used to define a 'hypermutator' phenotype. ${ }^{7} 8$ Tumors with a large number of somatic mutations 
(commonly $>10$ mutations per $\mathrm{Mb}$ ) and/or mutations in mismatch repair (MMR) or DNA polymerase genes are referred to as hypermutated tumors. ${ }^{78}$ A hypermutator phenotype is seen across different tumor types with varying frequencies and can either happen de novo or as a result of exposure to alkylating agents. ${ }^{9}$ Marked response to CPIs have been reported in two patients with corticotroph PC, including one tumor with a hypermutator phenotype. ${ }^{1011}$

Here, we report four cases of refractory PC treated with pembrolizumab. Two out of four patients had a radiographic and hormonal response to CPI therapy.

\section{METHODS}

In this open-label, phase II trial, patients with advanced rare cancers whose tumors had progressed within the previous 6 months received pembrolizumab $200 \mathrm{mg}$ intravenously every 21 days. ${ }^{12}$ Immune-Related Response Evaluation Criteria In Solid Tumors (irRECIST) ${ }^{13}$ was applied to report responses. The adverse events were graded according to the National Cancer Institute Common Terminology Criteria for Adverse Events V.4.03. PD-L1 staining was performed by Qualtek using Merck 22C3 antibody for PD-L1. Based on the percentage and intensity of membrane staining, an $\mathrm{H}$-score from 0 to 300 was assigned to tumor samples. ${ }^{14}$ To measure TILs, we performed a morphological assessment of H\&E-stained sections to determine the overall average abundance value of TILs within tumor nests for the whole sample, using a scale of 0 (absent) to 3. High TIL score was defined as a TIL density score $\geq 2$. PD-L1 and TIL scores were completed by a board-certified pathologist.

The data cut-off for report of these data was May 4, 2020. It was planned to enroll 15 patients in this cohort; however, due to rarity of $\mathrm{PC}$ and resultant slow accrual, we decided to report on the current four patients.

\section{RESULTS}

We treated four patients with PC with pembrolizumab (table 1). All patients tolerated the treatment well with mild adverse events (table 2 ).

\section{Patient 1}

A white male in his mid-30s presented with visual field deficits and Cushingoid features. Imaging revealed a pituitary macroadenoma and he underwent tumor resection. Histological examination demonstrated a corticotroph adenoma. The patient received radiotherapy for residual disease. In the following 3 years, he underwent another resection and two additional courses of focal radiotherapy for progressive disease. Fifty-four months after the initial diagnosis, he reported abdominal fullness and discomfort and was found to have liver lesions on imaging. Biopsy of a liver lesion demonstrated neuroendocrine carcinoma immunoreactive for ACTH and synaptophysin, consistent with metastatic corticotroph PC. He was treated with standard doses of temozolomide for 16 cycles and CAPTEM for 2 cycles before undergoing right orbital exenteration and additional focal radiotherapy for symptomatic progressive right orbital disease. The resected tissue was analyzed by next-generation sequencing (NGS) using a 400-gene panel and demonstrated a hypermutator phenotype with 76 individual gene mutations, including MSH2 and MSH6 mutations. He subsequently received local treatment to the liver lesions (percutaneous microwave frequency ablation) and portacaval lymphadenopathy (radiotherapy), bilateral adrenalectomy for uncontrolled Cushing disease and an additional eight cycles of standard dose temozolomide and four cycles of CAPTEM without response. Given a somatic FGFR4 mutation, he was enrolled into a phase I trial of an FGFRtargeted treatment and completed two cycles. His intracranial and extracranial disease continued to progress, requiring radiotherapy to the liver, resection of disease adjacent to the left optic nerve and further intracranial radiotherapy to a suspected right temporal tumor, eventually complicated by radiation necrosis.

Ultimately, after six lines of systemic chemotherapy, the patient was enrolled in the phase II trial of pembrolizumab. Staging after two cycles of pembrolizumab demonstrated resolution of the lesions in the middle cranial fossa and prepontine cisterns and significant improvement of disease in the bilateral sphenoid and posterior ethmoid sinuses (figure 1A\&B). In addition, there was an interval decrease in the retroperitoneal adenopathy; the size of the liver metastases remained stable. His overall best radiographic response was partial response $(60 \%$ per irRECIST criteria), which persists 42 and 22 months after the first and last dose of pembrolizumab, respectively. Plasma ACTH levels were 48 and $85 \mathrm{pg} / \mathrm{mL}$ (range $0-46)$ prior to initiation of pembrolizumab and became undetectable after treatment, where it remains to date (figure 1C). He completed 29 cycles of pembrolizumab and tolerated it very well. His subsequent treatments have been geared toward the management of radiation necrosis. He has remained cancer free 42 months after his first dose of pembrolizumab, and he is alive 118 months after the diagnosis of PC. Analysis of liver tumor tissue obtained prior to trial enrollment demonstrated negative PD-L1 staining and a TIL score of 2. He is currently in his early 50s, and his Eastern Cooperative Oncology Group (ECOG) status is 2, mainly due to functional blindness secondary to progressive radiation-induced optic neuropathy in the left eye.

\section{Patient 2}

A Hispanic woman in her early 20s presented with Cushingoid features and was found to have a pituitary macroadenoma. Resection of the mass revealed a necrotic tumor consistent with infarcted/necrotic pituitary adenoma and strong ACTH staining. Eight months later, the patient required additional resection of the corticotroph adenoma. She received stereotactic radiosurgery for residual disease; a year later, she required 


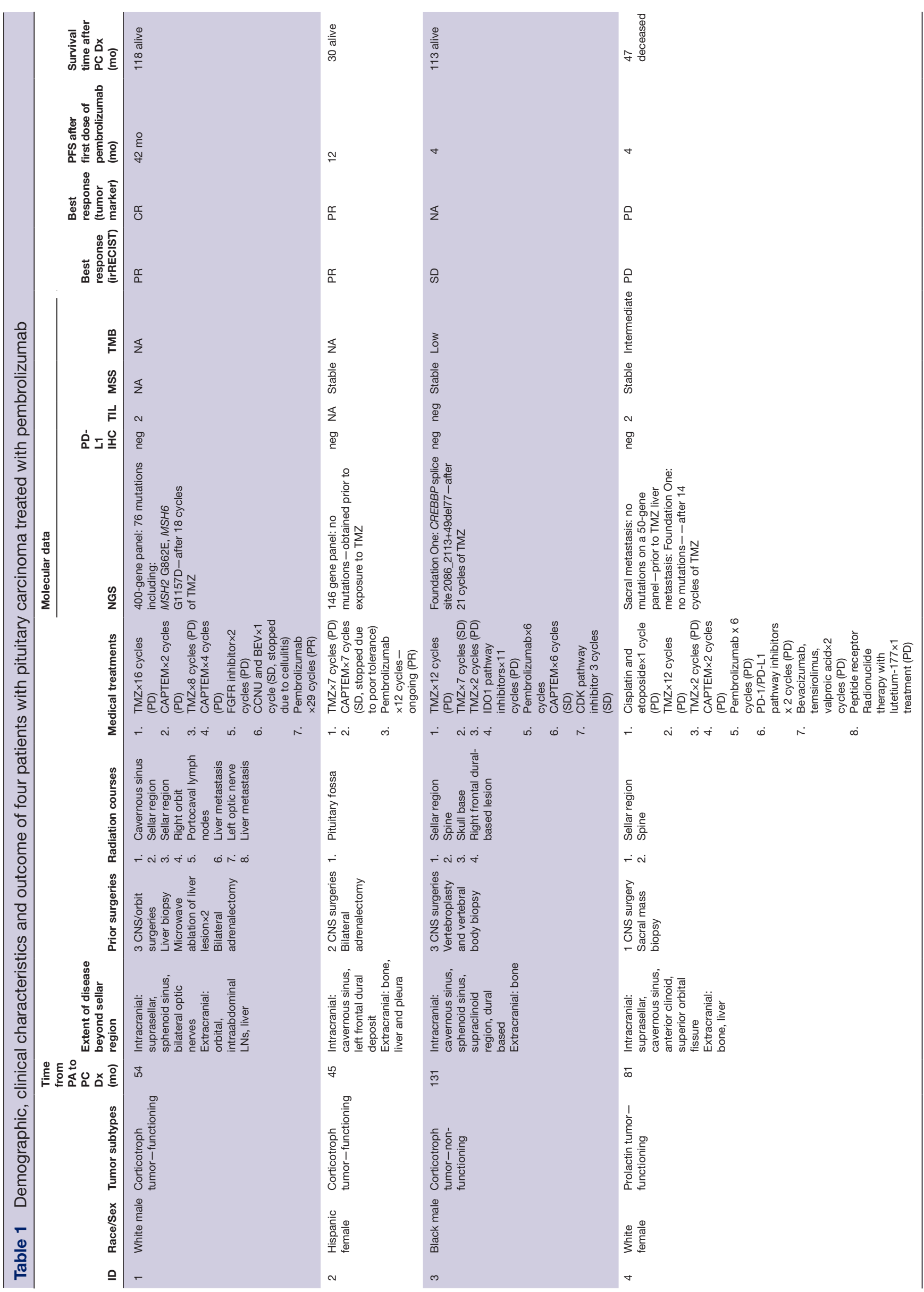




\begin{tabular}{|c|c|c|c|c|c|}
\hline \multirow{3}{*}{ 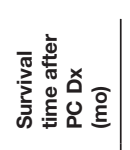 } & \multirow{3}{*}{ 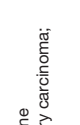 } & \\
\hline & & \multicolumn{4}{|c|}{ Table 2 Adverse events } \\
\hline & & ID & Event & Grade & Attribution \\
\hline \multirow{4}{*}{ 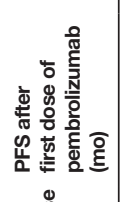 } & \multirow{6}{*}{ 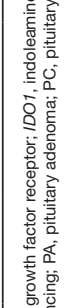 } & 1 & Fever & 2 & Unlikely \\
\hline & & 1 & Fatigue & 1 & Definite \\
\hline & & 1 & Maculopapular rash & 1 & Possible \\
\hline & & 1 & Anorexia & 1 & Possible \\
\hline \multirow{4}{*}{ 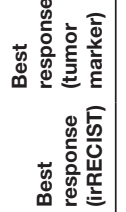 } & & 1 & Myalgia & 1 & Possible \\
\hline & & 1 & Nausea & 1 & Possible \\
\hline & 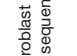 & 2 & Fatigue & 2 & Possible \\
\hline & 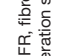 & $3-4$ & Fatigue & 1 & Possible \\
\hline
\end{tabular}

bilateral adrenalectomy for refractory Cushing disease. She continued to have rising ACTH levels, as high as $20000 \mathrm{pg} / \mathrm{mL}$, associated with severe skin hyperpigmentation. Treatment with the somatostatin analog pasireotide resulted in only a transient clinical response. Due to persistently rising ACTH levels, reaching a peak of nearly $85000 \mathrm{pg} / \mathrm{mL}$, restaging was performed 45 months after initial diagnosis. MRI of the brain showed stable disease, but MRI of the spine showed numerous lesions throughout the axial skeleton, compatible with metastatic disease. She was started on temozolomide and her ACTH decreased to the $1000 \mathrm{pg} / \mathrm{mL}$ range. She received 14 cycles of temozolomide-containing regimens but had to stop due to grade 2 fatigue and nausea. Ultimately, after two lines of systemic therapy, she was enrolled in the pembrolizumab trial and achieved a partial unconfirmed radiographic response (32\% reduction in sum of liver lesions per irRECIST) after 15 cycles without significant adverse events as of the reporting of this manuscript. After an initial rise in ACTH levels after commencing pembrolizumab (from 2000 to $27460 \mathrm{pg}$ / $\mathrm{mL}$ over a 3-month period), her ACTH levels started to decline 3 weeks after the peak and was associated with improved skin coloration (figure 2). Her tumor tissue prior to exposure to temozolomide (studied with a 146gene NGS panel) showed no mutations and stable microsatellite status (MSS); PD-L1 staining was negative. No additional tumor samples were obtained after exposure to temozolomide. She is currently in her late 20 s, and her ECOG status is 1.

\section{Patient 3}

A black man in his late teens presented with visual field deficits on a routine eye examination, which led to the diagnosis of pituitary macroadenoma. Resection was performed, and histology was consistent with a corticotroph adenoma, which was clinically non-functioning (a silent corticotroph adenoma). He received radiotherapy to the sellar region followed by tumor debulking. Approximately 10 years after the initial diagnosis, he presented with severe back pain. Imaging revealed complete replacement of $\mathrm{T} 8$ by a lesion and additional small lesions in multiple other thoracic and lumbar vertebrae. Vertebroplasty was performed and T8 biopsy revealed PC. He 

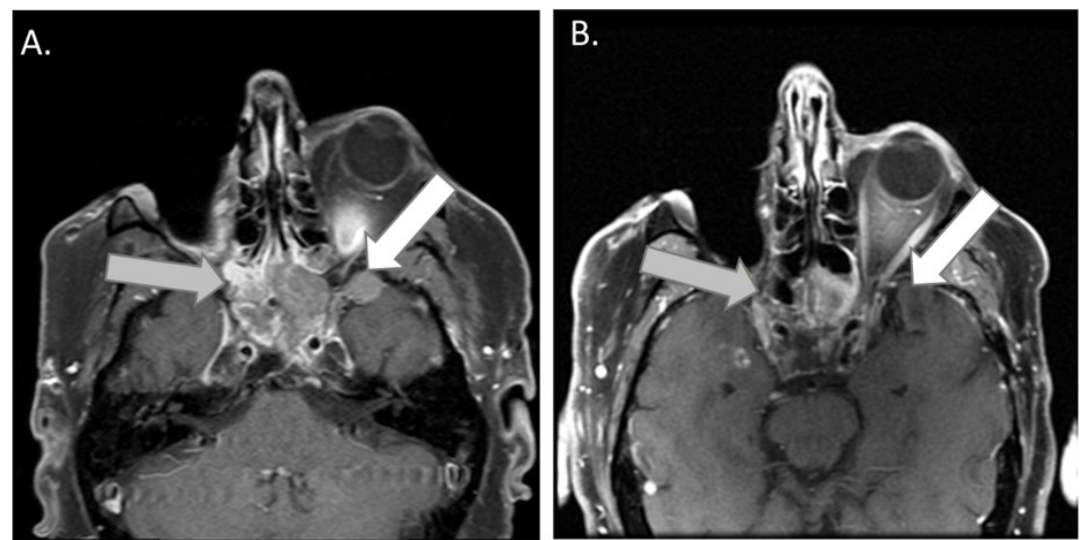

C.

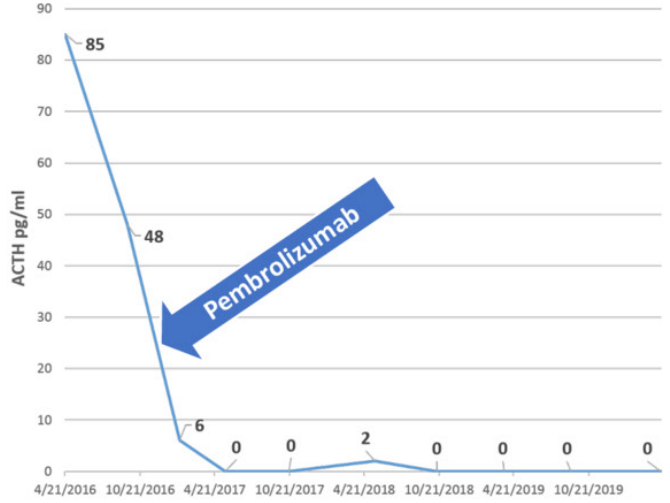

Dates of ACTH Measurements Before and After Pembrolizumab

Figure 1 Response to pembrolizumab in patient 1. (A) T1 postcontrast MRI obtained prior to pembrolizumab treatment demonstrates a lobulated enhancing mass within the sphenoid sinus and posterior ethmoid air cells (gray arrow) with extension to the anterior and inferior aspects of the left temporal lobe (white arrow). (B) MRI of the brain after two cycles of pembrolizumab demonstrates significant improvement of the enhancing mass in the cavernous sinus and resolution of the nodule extending to the left temporal lobe. (C) The adrenocorticotropic hormone (ACTH) level became undetectable after treatment with pembrolizumab. (Reference ranges for ACTH are 0-46 pg/mL (before 4/19/18) and 7-63 pg/mL (after 04/19/18); ACTH levels are listed as ' 0 ' if the reported value was less than the lower limit of detection for the assay.)

underwent radiotherapy to the skull base and spine and transsphenoidal debulking, followed by two courses of temozolomide (nine cycles). Due to progressive disease, he had a fourth tumor resection. Foundation One

Figure 2. Patient 2's hormonal response to pembrolizumab

A.

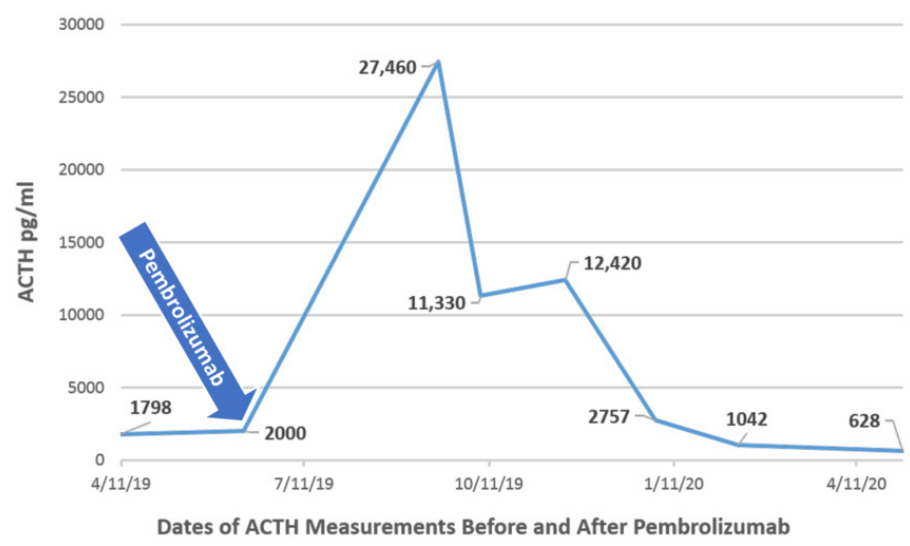

Figure 2 Clinical response in patient 2. (A) Adrenocorticotropic hormone (ACTH) levels became significantly elevated after starting pembrolizumab and then gradually declined but have not yet nadired as of the last evaluation. (B) Overview of the patient's skin pigmentation changes. (i) Two and a half years before the diagnosis of pituitary carcinoma, the patient had normal skin coloration and an ACTH level of $160 \mathrm{pg} / \mathrm{mL}$. (ii) At diagnosis of pituitary carcinoma, when the ACTH was $84851 \mathrm{pg} / \mathrm{mL}$, the patient had become remarkably hyperpigmented. Ten months after treatment with pembrolizumab commenced and when the ACTH was $628 \mathrm{pg} / \mathrm{mL}$ (iv), her skin pigmentation was noticeably lighter compared with pictures obtained 6 months earlier (iii), after 4 months of therapy, when her ACTH was $>11000 \mathrm{pg} / \mathrm{mL}$. (Reference ranges for ACTH are 0-46 pg/mL (before 4/19/18) and $7-63 \mathrm{pg} / \mathrm{mL}$ (after 04/19/18).). molecular testing showed KRAS amplification, a CREBBP splice site mutation, multiple DNA variants of uncertain significance, low tumor mutational burden, stable MSS and negative PD-L1 immunohistochemical staining. One 
year later, he had progression of dural-based lesions, and he enrolled in a phase I trial of treatment targeting the indoleamine 2,3 dioxygenase 1 pathway, with a continued slow increase in central skull base lesions. Ultimately, he enrolled in the pembrolizumab trial and completed six cycles, with a best radiographic response of stable disease for 4 months. Due to progressive partial palsy of the right oculomotor nerve and gradual progression of the skull base disease infiltrating the right cavernous sinus (without progressive disease per irRECIST), he came off study. He is currently in his late $30 \mathrm{~s}$, and his ECOG status is 1 .

\section{Patient 4}

A white Middle Eastern woman in her early 50s was diagnosed with a macroprolactinoma that did not respond to conventional dopamine agonist therapy. She was found to have osseous metastasis to the spine 81 months after diagnosis of the lactotroph adenoma. NGS with a 134-gene panel on a sacral metastasis showed no mutations. She received one cycle of cisplatin and etoposide followed by palliative radiotherapy to the thoracic and lumbosacral spine. She then received 12 cycles of standard dose temozolomide. She had an increase in PRL levels and new tumors in the liver. She was re-challenged with two additional cycles of temozolomide and then switched to CAPTEM for two cycles, but she experienced enlarging lesions in bone and liver and an ongoing rise in PRL levels. She then enrolled in the pembrolizumab trial. Her overall response was progressive disease per irRECIST. During pembrolizumab therapy, her PRL rose from 2139 to $5879 \mathrm{ng} / \mathrm{mL}$ (range $4.8-23.3$ ). Tissue analysis prior to trial enrollment showed negative PD-L1 staining and a TIL score of 2. A subsequent liver biopsy showed stable MSS and intermediate tumor mutational burden. She died of progressive disease 46 months after the diagnosis of PC.

\section{DISCUSSION}

CPIs have changed the outcomes of many patients with cancer, and their approvals by the US Food and Drug Administration (FDA) are on the rise, including the first tumor-agnostic approval for pembrolizumab for microsatellite instability-high or mismatch repair deficient solid tumors. ${ }^{15}$ Despite small numbers of patients, our current report of four cases, which supplements four previously published cases, ${ }^{1011}$ provides the basis for further defining the role of CPIs in the treatment of PC.

In this study, we describe the positive radiographic and hormonal responses to pembrolizumab in two patients, both of whom had ACTH-secreting PC refractory to prior lines of systemic therapy. Patient 1's response has been durable for 42 months. Genomic analysis of tumor tissue obtained from this patient after 16 cycles of temozolomide demonstrated mutations in 76 genes of a 400-gene panel, including MSH2 G862E and MSH6 G1157D, indicative of a hypermutator phenotype. MSH2 and MSH6 are essential mismatch repair (MMR) system components that detect base-base mismatches or insertion/deletion loops and perform error removal and DNA resynthesis. ${ }^{16}$ MMR-deficient tumors have a large proportion of mutant neoantigens that result in in vivo expansion of neoantigen-specific T-cell clones that are reactive to the mutant neopeptides within the tumor and therefore increase responsiveness to CPI. ${ }^{17}$ Patient 2's tumor after exposure to temozolomide was not sampled so its mutation status postchemotherapy is unknown. Interestingly, the two tumors that did not demonstrate a response (patients 3 and 4) did not demonstrate a hypermutator phenotype after exposure to temozolomide.

In patient 1 , hypermutation might have been due to exposure to temozolomide; however, the patient's temozolomide-naïve tissue is not available for NGS testing for comparison. Lin et al reported a case of marked response to nivolumab (anti-PD-1) and ipilimumab (antiCTLA-4) in a corticotroph PC. ${ }^{11}$ The authors performed genomic analysis of the tumor before and after exposure to temozolomide and demonstrated the emergence of a hypermutator phenotype after temozolomide exposure. Conversely, Caccese et al reported rapid disease progression of a corticotroph PC with MMR deficiency treated with pembrolizumab. ${ }^{18}$ This case, however, in contradistinction to the first published case and our two responders, was complicated by ongoing hypercortisolism, which may have attenuated the beneficial effects of CPI therapy. A correlation between MMR deficiency and high mutational burden has been reported in gliomas, ${ }^{19}$ but this correlation in PC remains to be identified.

Elevated PD-L1 expression is a predictor of response to anti-PD-1/PD-L1 therapies in some tumors, and companion PD-L1 diagnostic expression assays have been approved by the FDA. One study evaluated PD-L1 RNA and protein expression in 48 pituitary tumors and demonstrated increased expression of PD-L1 RNA and protein in functioning (somatotroph and lactotroph) pituitary adenomas compared with clinically non-functioning (null cell and gonadotroph) adenomas. ${ }^{20}$ Elevated PD-L1 expression was positively correlated with increased TILs and was enriched in primary tumors compared with recurrent tumors. In our case series, all patients had negative PD-L1 staining, including our patient with a durable response, indicating that PD-L1 staining may not be an indicator of response to CPIs in PC. However, this needs to be confirmed in larger studies. Two of four patients with available pretrial TIL data had TIL scores of 2 (patient 1 (responder) and patient 4 (non-responder)). It is not yet clear if PD-L1 expression and/or the presence of TILs are predictors of response to CPIs in pituitary tumors.

The composition of various types of immune cells within the tumor microenvironment and their interaction with tumor cells play an important role in the ability of CPIs to mount immune responses against pituitary tumor cells. One study that analyzed CD11b-expressing cells (expressed on monocytes, neutrophils, natural killer cells, granulocytes and macrophages) in 16 non-functioning pituitary tumors demonstrated that a higher proportion of $\mathrm{CD} 11^{+}$ 
cells $(>10 \%)$ was associated with a more expansile and mitotically active tumor. ${ }^{21}$ This study also demonstrated that tumors invading the cavernous sinus had a greater presence of immunosuppressive macrophages (M2) than did non-invasive tumors (M2/M1 ratio > 1 vs $\mathrm{M} 2 / \mathrm{M} 1$ ratio $<1)$. The correlation between increased number of immunosuppressive M2 macrophages and invasive behavior has been described in many cancer types. ${ }^{22}$ Another study evaluated different immune cell populations in 35 human pituitary tumors and demonstrated increased number of $\mathrm{CD} 8^{+}$macrophages in sparsely granulated $\mathrm{GH}$ and null cell tumors compared with densely granulated $\mathrm{GH}$ and corticotroph tumors. ${ }^{23}$ In addition, the number of $\mathrm{CD} 68^{+}$ macrophages has been shown to positively correlate with tumor size and local invasion. ${ }^{24}$ Evaluating the immune cell composition of tumors is needed for successful clinical development of CPI therapy in pituitary tumors. Our study did not include an analysis of the tumor immune cell composition.

Drugs that target CTLA-4 are more likely than other CPIs to cause hypophysitis and pituitary dysfunction, and their combination with medications that target PD-1 further heightens that risk. ${ }^{25}$ Isolated ACTH deficiency can also occur after use of single-agent PD-1 inhibitors. ${ }^{26}$ Importantly, although recovery of anterior pituitary hormone secretion can be seen, central adrenal insufficiency is usually permanent. ${ }^{27}$ This raises the question as to whether there may be an increased intrinsic susceptibility of corticotroph tumors to immune checkpoint inhibition, as observed in our study (patients 1 and 2) as well as the two other previously published cases. ${ }^{1011}$

It has also been hypothesized that pituitary tumors may particularly be sensitive to anti-CTLA- 4 antibodies due to the ectopic expression of CTLA-4 on pituitary tumors, which may be in part responsible for higher rates of hypophysitis after anti-CTLA-4 versus anti-PD-1 therapy. Our case of a responder treated with pembrolizumab monotherapy demonstrates that PD-1 blockade alone may be sufficient to induce a remarkable response in a hypermutated PC. It would be interesting to know if patients without a hypermutator phenotype who did not respond to PD-1 blockade alone would have a response to dual anti-CTLA-4 and anti-PD-1 therapy. The ongoing phase II trials of nivolumab and ipilimumab in patients with aggressive pituitary tumors (NCT04042753 and NCT02834013) have the potential to address this question.

\section{CONCLUSION}

Determining the efficacy of CPIs is difficult in rare tumors such as PC due to the challenges involved with conducting large clinical trials for an orphan disease. Here, we report four patients with PC treated with pembrolizumab-in whom two partial responses were seen in patients with ACTH-secreting PC. Further studies are needed to determine if the response to CPIs in patients with $\mathrm{PC}$ is unique to corticotroph tumors and to further clarify how the response correlates to the mutational status of the tumor and the tumor microenvironment.

\section{Twitter Nazanin Majd @NazMajd and Aung Naing @AnaingMD}

Acknowledgements Editorial support was provided by Bryan Tutt in Scientific Publications Services, Research Medical Library at the University of Texas MD Anderson Cancer Center. The authors would like to thank Bettzy Stephen, Jing Gong and Saria Kahn from the Department of Investigational Cancer Therapeutics at the University of Texas MD Anderson Cancer Center for their support. The authors would also like to thank the study participants and their families.

Contributors Manuscript preparation: NM, SGW, AN. Concept development: FJ, SF, ANTrial design and funding: FJ, SF, AN. Patient enrollment: NM, SGW, FJ, SF, MP-P, CK-M, SMR, IEMcC, AN. Data collection: NM, SGW, MX, AA, AN. Manuscript review and editing: NM, SGW, FJ, SF, MP-P, MX, AA, CK-M, SMR, IEMCC, AN.

Funding Merck Sharp \& Dohme Corp., a subsidiary of Merck \& Co., Inc. provided the study drug and funded this investigator-initiated trial.

Competing interests No, there are no competing interests.

Patient consent for publication Obtained.

Ethics approval The protocol was approved by the University of Texas MD Anderson Cancer Center Institutional Review Board. All subjects provided written informed consent when they enrolled in the trial, and patient 2 gave written informed consent for publication of her photographs.

Provenance and peer review Not commissioned; externally peer reviewed.

Data availability statement Data are available on reasonable request. All relevant data and material are available at MD Anderson Cancer Center on request.

Open access This is an open access article distributed in accordance with the Creative Commons Attribution Non Commercial (CC BY-NC 4.0) license, which permits others to distribute, remix, adapt, build upon this work non-commercially, and license their derivative works on different terms, provided the original work is properly cited, appropriate credit is given, any changes made indicated, and the use is non-commercial. See http://creativecommons.org/licenses/by-nc/4.0/.

\section{ORCID iDs}

Nazanin Majd http://orcid.org/0000-0001-6451-544X

Aung Naing http://orcid.org/0000-0002-4803-8513

\section{REFERENCES}

1 Lloyd RV, Osamura RY, Klöppel G, et al. Who classification of tumours of endocrine organs. 4th edn. Lyon: IARC Press, 2017.

2 Buurman H, Saeger W. Subclinical adenomas in postmortem pituitaries: classification and correlations to clinical data. Eur $J$ Endocrinol 2006;154:753-8.

3 Daly AF, Tichomirowa MA, Beckers A. The epidemiology and genetics of pituitary adenomas. Best Pract Res Clin Endocrinol Metab 2009;23:543-54.

4 Raverot G, Burman P, McCormack A, et al. European Society of endocrinology clinical practice guidelines for the management of aggressive pituitary tumours and carcinomas. Eur $J$ Endocrinol 2018;178:G1-24.

5 Zacharia BE, Gulati AP, Bruce JN, et al. High response rates and prolonged survival in patients with corticotroph pituitary tumors and refractory Cushing disease from capecitabine and temozolomide (CAPTEM): a case series. Neurosurgery 2014;74:E447-55. discussion E55.

6 Darvin P, Toor SM, Sasidharan Nair V, et al. Immune checkpoint inhibitors: recent progress and potential biomarkers. Exp Mol Med 2018;50:1-11.

7 Campbell BB, Light N, Fabrizio D, et al. Comprehensive analysis of hypermutation in human cancer. Cell 2017;171:1042-56.

8 Kandoth C, McLellan MD, Vandin F, et al. Mutational landscape and significance across 12 major cancer types. Nature 2013;502:333-9.

9 Kamiya-Matsuoka C, Metrus NR, Shaw KR, et al. The natural course of hypermutator gliomas. Journal of Clinical Oncology 2018;36:2014.

10 Duhamel C, Ilie MD, Salle H, et al. Immunotherapy in corticotroph and lactotroph aggressive tumors and carcinomas: two case reports and a review of the literature. J Pers Med 2020;10. doi:10.3390/ jpm10030088. [Epub ahead of print: 1308 2020].

11 Lin AL, Jonsson P, Tabar V, et al. Marked response of a hypermutated ACTH-secreting pituitary carcinoma to ipilimumab and nivolumab. J Clin Endocrinol Metab 2018;103:3925-30. 
12 Naing A, Meric-Bernstam F, Stephen B, et al. Phase 2 study of pembrolizumab in patients with advanced rare cancers. $J$ Immunother Cancer 2020;8:e000347.

13 Nishino M, Tirumani SH, Ramaiya NH, et al. Cancer immunotherapy and immune-related response assessment: the role of radiologists in the new arena of cancer treatment. Eur J Radiol 2015;84:1259-68.

14 Dolled-Filhart M, Locke D, Murphy T, et al. Development of a prototype immunohistochemistry assay to measure programmed death ligand-1 expression in tumor tissue. Arch Pathol Lab Med 2016;140:1259-66.

15 Marabelle A, Le DT, Ascierto PA, et al. Efficacy of pembrolizumab in patients with Noncolorectal high microsatellite Instability/Mismatch repair-deficient cancer: results from the phase II KEYNOTE-158 study. J Clin Oncol 2020;38:1-10.

16 Kunkel TA, Erie DA. Eukaryotic mismatch repair in relation to DNA replication. Annu Rev Genet 2015;49:291-313.

17 Le DT, Durham JN, Smith KN, et al. Mismatch repair deficiency predicts response of solid tumors to PD-1 blockade. Science 2017;357:409-13.

18 Caccese M, Barbot M, Ceccato F, et al. Rapid disease progression in patient with mismatch-repair deficiency pituitary ACTH-secreting adenoma treated with checkpoint inhibitor pembrolizumab. Anticancer Drugs 2020;31:199-204.

19 Hodges TR, Ott M, Xiu J, et al. Mutational burden, immune checkpoint expression, and mismatch repair in glioma: implications for immune checkpoint immunotherapy. Neuro Oncol 2017;19:1047-57.
20 Mei Y, Bi WL, Greenwald NF, et al. Increased expression of programmed death ligand 1 (PD-L1) in human pituitary tumors. Oncotarget 2016;7:76565-76.

21 Yagnik G, Rutowski MJ, Shah SS, et al. Stratifying nonfunctional pituitary adenomas into two groups distinguished by macrophage subtypes. Oncotarget 2019;10:2212-23.

22 Condeelis J, Pollard JW. Macrophages: obligate partners for tumor cell migration, invasion, and metastasis. Cell 2006;124:263-6.

$23 \mathrm{Lu} \mathrm{J-Q,} \mathrm{Adam} \mathrm{B,} \mathrm{Jack} \mathrm{AS,} \mathrm{et} \mathrm{al.} \mathrm{Immune} \mathrm{cell} \mathrm{infiltrates} \mathrm{in}$ pituitary adenomas: more macrophages in larger adenomas and more T cells in growth hormone adenomas. Endocr Pathol 2015;26:263-72.

24 Knosp E, Steiner E, Kitz K, et al. Pituitary adenomas with invasion of the cavernous sinus space: a magnetic resonance imaging classification compared with surgical findings. Neurosurgery 1993;33:610-7. discussion 7-8.

25 Barroso-Sousa R, Barry WT, Garrido-Castro AC, et al. Incidence of endocrine dysfunction following the use of different immune checkpoint inhibitor regimens: a systematic review and metaanalysis. JAMA Oncol 2018;4:173-82.

26 Kitajima K, Ashida K, Wada N, et al. Isolated ACTH deficiency probably induced by autoimmune-related mechanism evoked with nivolumab. Jpn J Clin Oncol 2017;47:463-6.

27 Tan $\mathrm{MH}$, lyengar $\mathrm{R}$, Mizokami-Stout $\mathrm{K}$, et al. Spectrum of immune checkpoint inhibitors-induced endocrinopathies in cancer patients: a scoping review of case reports. Clin Diabetes Endocrinol 2019;5:1. 